PROCEEDINGS OF THE

AMERICAN MATHEMATICAL SOCIETY

Volume 139, Number 10, October 2011, Pages 3553-3560

S 0002-9939(2011)10755-X

Article electronically published on February 10, 2011

\title{
THE BISHOP-PHELPS-BOLLOBÁS THEOREM AND ASPLUND OPERATORS
}

\author{
R. M. ARON, B. CASCALES, AND O. KOZHUSHKINA \\ (Communicated by Nigel J. Kalton) \\ Dedicated to the memory of Nigel J. Kalton
}

\begin{abstract}
This paper deals with a strengthening of the Bishop-Phelps property for operators that in the literature is called the Bishop-Phelps-Bollobás property. Let $X$ be a Banach space and $L$ a locally compact Hausdorff space. We prove that if $T: X \rightarrow C_{0}(L)$ is an Asplund operator and $\left\|T\left(x_{0}\right)\right\| \approx\|T\|$ for some $\left\|x_{0}\right\|=1$, then there is a norm-attaining Asplund operator $S: X \rightarrow$ $C_{0}(L)$ and $\left\|u_{0}\right\|=1$ with $\left\|S\left(u_{0}\right)\right\|=\|S\|=\|T\|$ such that $u_{0} \approx x_{0}$ and $S \cong T$. As particular cases we obtain: (A) if $T$ is weakly compact, then $S$ can also be taken to be weakly compact; (B) if $X$ is Asplund (for instance, $X=c_{0}$ ), the pair $\left(X, C_{0}(L)\right)$ has the Bishop-Phelps-Bollobás property for all $L ;(\mathrm{C})$ if $L$ is scattered, the pair $\left(X, C_{0}(L)\right)$ has the Bishop-Phelps-Bollobás property for all Banach spaces $X$.
\end{abstract}

\section{INTRODUCTION}

In this paper we are concerned with the study of simultaneously approximating both operators and the points at which they almost attain their norms by normattaining operators and the points at which they attain their norms. Namely, we study what in recent literature has been called the Bishop-Phelps-Bollobás property. Using standard notation that we fix at the end of this introduction, this property is defined as:

Definition 1.1 (Acosta, Aron, García and Maestre, 1]). A pair of Banach spaces $(X, Y)$ is said to have the Bishop-Phelps-Bollobás property (BPBP) if for any $\varepsilon>0$ there are $\eta(\varepsilon)>0$ and $\beta(\varepsilon)>0$ with $\lim _{t \rightarrow 0} \beta(t)=0$, such that for all $T \in S_{L(X, Y)}$, if $x_{0} \in S_{X}$ is such that $\left\|T\left(x_{0}\right)\right\|>1-\eta(\varepsilon)$, then there are $u_{0} \in S_{X}$ and $S \in S_{L(X, Y)}$ satisfying

$$
\left\|S\left(u_{0}\right)\right\|=1,\left\|x_{0}-u_{0}\right\|<\beta(\varepsilon) \text { and }\|T-S\|<\varepsilon .
$$

Received by the editors July 23, 2010 and, in revised form, August 24, 2010.

2010 Mathematics Subject Classification. Primary 46B22; Secondary $47 \mathrm{~B} 07$.

Key words and phrases. Bishop-Phelps, Bollobás, fragmentability, Asplund operator, weakly compact operator, norm-attaining.

The research of the first author was supported in part by MICINN Project MTM2008-03211.

The research of the second author was supported by FEDER and MEC Project MTM200805396 and by Fundación Séneca (CARM), project 08848/PI/08.

The research of the third author was supported in part by U.S. National Science Foundation grant DMS-0652684. 
The above BPBP was motivated by the following result of Bollobás:

Theorem 1.2 (Bollobás, [5]). Given $\frac{1}{2}>\varepsilon>0$, if $x_{0} \in S_{X}$ and $x^{*} \in S_{X^{*}}$ are such that

$$
\left|1-x^{*}\left(x_{0}\right)\right|<\frac{\varepsilon^{2}}{2},
$$

then there are $u_{0} \in S_{X}$ and $y^{*} \in S_{X^{*}}$ such that

$$
y^{*}\left(u_{0}\right)=1,\left\|x_{0}-u_{0}\right\|<\varepsilon+\varepsilon^{2} \text { and }\left\|x^{*}-y^{*}\right\|<\varepsilon .
$$

Bollobás' result is indeed an observation about the classical Bishop-Phelps' theorem, 4, that in the words of its author "sharpen Bishop-Phelps' theorem and is applied to some problems about the numerical range of operators." Using Definition 1.1. Bollobás' Theorem 1.2 can be rephrased by saying that for every Banach space $X$ the pair $(X, \mathbb{R})$ has BPBP.

In [1, the authors described a number of cases of pairs $(X, Y)$ with BPBP. For instance, they proved that if $Y$ has property $(\beta)$, see [18, Definition 1.2], then $(X, Y)$ has BPBP for every Banach space $X$. Also, $\left(\ell^{1}, Y\right)$ has $\operatorname{BPBP}$ for $Y$ in a large class of Banach spaces that includes the finite-dimensional Banach spaces, uniformly convex Banach spaces, spaces $L_{1}(\mu)$ for a $\sigma$-finite measure $\mu$ and spaces $C(K)$. Although some particular results can be found in [1, Section 5] for pairs of the form $\left(\ell_{n}^{\infty}, Y\right)$ (for instance, $Y$ uniformly convex), the authors of [1] comment that their methods do not work for pairs of the form $\left(c_{0}, Y\right)$. Our aim here is to devise a method to study the Bishop-Phelps-Bollobás property that in particular addresses this question when $Y=C_{0}(L), L$ a locally compact Hausdorff space.

The following describes the contents of this paper. After fixing some standard notation, we recall in section 2 the notions of Asplund space and Asplund operator. In this section, we also prove a central technical result (Lemma 2.3) that will be used to prove our main result, Theorem 2.4. Theorem 2.4 establishes that if $T: X \rightarrow C_{0}(L)$ is an Asplund operator and $\left\|T\left(x_{0}\right)\right\| \approx\|T\|$ for some $\left\|x_{0}\right\|=1$, then there is a norm-attaining Asplund operator $S: X \rightarrow C_{0}(L)$ and $\left\|u_{0}\right\|=1$ with $\left\|S\left(u_{0}\right)\right\|=\|S\|=\|T\|$ such that $u_{0} \approx x_{0}$ and $S \approx T$.

Three consequences follow:

(A) If $T$ is weakly compact, then $S$ can also be taken to be weakly compact (see Corollary 2.5).

(B) If $X$ is Asplund, then the pair $\left(X, C_{0}(L)\right)$ has the BPBP for all $L$ (see Corollary 2.6).

(C) If $L$ is scattered, then the pair $\left(X, C_{0}(L)\right)$ has the BPBP for all $X$ (see Corollary 2.7].

We note that in Corollary 2.5 even the part of the density of norm-attaining weakly compact operators from $X$ to $C_{0}(L)$ in the family of weakly compact operators $\mathcal{W}\left(X, C_{0}(L)\right)$ seems to be new. Corollary 2.6] strengthens a result in [1], and Corollary 2.7 can be alternatively proved using a result in [1].

Notation and terminology. All vector spaces in this paper are assumed to be real. By $X$ and $Y$ we always denote Banach spaces. $B_{X}$ and $S_{X}$ are the closed unit ball and the unit sphere of $X$, respectively. $X^{*}$ (resp. $X^{* *}$ ) stands for the topological dual (resp. bidual) of $X$. The weak topology is denoted by $w$, and $w^{*}$ is the weak* topology in the dual. $L(X, Y)$ denotes the spaces of bounded linear operators from $X$ to $Y$ endowed with its usual norm. 
The letters $K$ and $L$ are reserved to denote compact and locally compact Hausdorff spaces respectively. $C(K)$ (resp. $C_{0}(L)$ ) denotes the space of real-valued continuous functions (resp. continuous functions vanishing at infinity) on $K$ (resp. on $L$ ) endowed with the standard sup norm, which is simply denoted by $\|f\|:=$ $\sup \{|f(s)|: s \in K\}$. As usual, given $s \in L$ we denote by $\delta_{s}: C_{0}(L) \rightarrow \mathbb{R}$ the Dirac measure at $s$ given by $\delta_{s}(f)=f(s), f \in C_{0}(L)$.

\section{Asplund spaCes And Bishop-Phelps-BollobÁs theOrem}

The Banach space $X$ is called an Asplund space if, whenever $f$ is a convex continuous function defined on an open convex subset $U$ of $X$, the set of all points of $U$ where $f$ is Fréchet differentiable is a dense $G_{\delta}$-subset of $U$. This definition is due to Asplund [3] under the name strong differentiability space. Asplund spaces have been used profusely since they were introduced. The versatility of this concept is in part explained by its multiple characterizations via topology or measure theory, as for instance in the following:

Theorem 2.1. Let $X$ be a Banach space. Then the following conditions are equivalent:

(i) $X$ is an Asplund space;

(ii) every $w^{*}$-compact subset of $\left(X^{*}, w^{*}\right)$ is fragmented by the norm;

(iii) each separable subspace of $X$ has separable dual;

(iv) $X^{*}$ has the Radon-Nikodým property.

For the notion of the Radon-Nikodým property we refer to 6 , 8 . The equivalence (iii) $\Leftrightarrow$ (iv) is due to Stegall [20, (i) $\Leftrightarrow$ (ii) $\Rightarrow$ (iii) can be found in the paper by Namioka and Phelps [14, and (iii) $\Rightarrow$ (ii) is due again to Stegall [21]. Recall that a subset $C$ of $\left(X^{*}, w^{*}\right)$ is said to be fragmented by the norm if for each non-empty subset $A$ of $C$ and for each $\varepsilon>0$ there exists a non-empty $w^{*}$-open subset $U$ of $X^{*}$ such that $U \cap A \neq \emptyset$ and $\|\cdot\|-\operatorname{diam}(U \cap A) \leq \varepsilon$, [13]. We note that if $C$ is $w^{*}$-compact convex, then $C$ is fragmented by the norm if, and only if, $C$ has the Radon-Nikodým property; see [6, Theorem 4.2.13].

An operator $T \in L(X, Y)$ is said to be an Asplund operator if it factors through an Asplund space, i.e., if there are an Asplund space $Z$ and operators $T_{1} \in L(X, Z)$, $T_{2} \in L(Z, Y)$ such that $T=T_{2} \circ T_{1}$; see [10, 22]. Note that every weakly compact operator $T \in \mathcal{W}(X, Y)$ factors through a reflexive Banach space, see [7], and hence $T$ is an Asplund operator.

Lemma 2.3 isolates the technicalities that we need to prove our main result, Theorem 2.4 In the proof of the lemma, we use that Theorem 1.2 easily yields the following result.

Remark 2.2. Given $\frac{1}{2}>\varepsilon>0$, if $x_{0} \in S_{X}$ and $x^{*} \in S_{X^{*}}$ are such that

$$
\left|x^{*}\left(x_{0}\right)\right|>1-\frac{\varepsilon^{2}}{4},
$$

then there are $u_{0} \in S_{X}$ and $y^{*} \in S_{X^{*}}$ such that

$$
\left|y^{*}\left(u_{0}\right)\right|=1,\left\|x_{0}-u_{0}\right\|<\varepsilon \text { and }\left\|x^{*}-y^{*}\right\|<\varepsilon .
$$

Recall that a subset $B \subset B_{Y}^{*}$ is said to be 1-norming if

$$
\|y\|=\sup _{b^{*} \in B}\left|b^{*}(y)\right|
$$


for every $y \in Y$. Recall that if $T \in L(X, Y)$, then its adjoint $T^{*} \in L\left(Y^{*}, X^{*}\right)$ is also $w^{*}$-to- $w^{*}$ continuous.

Lemma 2.3. Let $T: X \rightarrow Y$ be an Asplund operator with $\|T\|=1$, let $\frac{1}{2}>\varepsilon>0$ and choose $x_{0} \in S_{X}$ such that

$$
\left\|T\left(x_{0}\right)\right\|>1-\frac{\varepsilon^{2}}{4}
$$

For any given 1-norming set $B \subset B_{Y^{*}}$, if we write $M:=T^{*}(B)$, then there are:

(a) a $w^{*}$-open set $U \subset X^{*}$ with $U \cap M \neq \emptyset$ and

(b) points $y^{*} \in S_{X^{*}}$ and $u_{0} \in S_{X}$ with $\left|y^{*}\left(u_{0}\right)\right|=1$ such that

$$
\left\|x_{0}-u_{0}\right\|<\varepsilon \text { and }\left\|z^{*}-y^{*}\right\|<3 \varepsilon \text { for every } z^{*} \in U \cap M \text {. }
$$

Proof. Observe first that if $T$ is an Asplund operator, then its adjoint $T^{*}$ sends the unit ball of $Y^{*}$ into a $w^{*}$-compact subset of $\left(X^{*}, w^{*}\right)$ that is norm fragmented. Indeed, if $T=T_{2} \circ T_{1}$ is a factorization through the Asplund space $Z$ for $T$, then its adjoint $T^{*}$ factors through $Z^{*}$ :

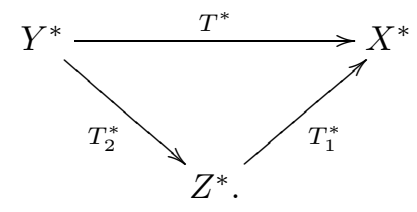

Since $T_{2}^{*}$ is $w^{*}$ - $w^{*}$ continuous, $T_{2}^{*}\left(B_{Y^{*}}\right)$ is a $w^{*}$-compact subset of $Z^{*}$, and we can now appeal to Theorem 2.1 to conclude that $T_{2}^{*}\left(B_{Y^{*}}\right) \subset\left(Z^{*}, w^{*}\right)$ is fragmented by the norm of $Z^{*}$. On the other hand, $T_{1}^{*}: Z^{*} \rightarrow X^{*}$ is norm-to-norm and $w^{*}$ $w^{*}$ continuous, and therefore it sends the fragmented $w^{*}$-compact set $T_{2}^{*}\left(B_{Y^{*}}\right) \subset$ $\left(Z^{*}, w^{*}\right)$ onto the $w^{*}$-compact set $T^{*}\left(B_{Y^{*}}\right) \subset\left(X^{*}, w^{*}\right)$ that is fragmented by the norm of $X^{*}$, see [13, Lemma 2.1], and our observation is proved. (Alternatively, the observation can be proved using [22, Theorem 2.11] and [6, Theorem 4.2.13].)

Now we really start the proof of the lemma. Use that $B \subset B_{Y^{*}}$ is 1-norming and pick $b_{0}^{*} \in B$ such that

$$
\left|T^{*}\left(b_{0}^{*}\right)\left(x_{0}\right)\right|=\left|b_{0}^{*}\left(T\left(x_{0}\right)\right)\right|>1-\frac{\varepsilon^{2}}{4} .
$$

Defining $U_{1}=\left\{x^{*} \in X^{*}:\left|x^{*}\left(x_{0}\right)\right|>1-\frac{\varepsilon^{2}}{4}\right\}$, we have that

$$
T^{*}\left(b_{0}^{*}\right) \in U_{1} \cap M \subset T^{*}\left(B_{Y^{*}}\right) \subset B_{X^{*}} .
$$

Since $T^{*}\left(B_{Y^{*}}\right)$ is fragmented and $U_{1} \cap M$ is non-empty, there exists a $w^{*}$-open set $U_{2} \subset X^{*}$ such that $\left(U_{1} \cap M\right) \cap U_{2} \neq \emptyset$ and

$$
\|\cdot\| \text { - diam }\left(\left(U_{1} \cap M\right) \cap U_{2}\right) \leq \varepsilon .
$$

Let $U:=U_{1} \cap U_{2}$ and fix $x_{0}^{*} \in U \cap M$. We have

$$
1 \geq\left\|x_{0}^{*}\right\| \geq\left|x_{0}^{*}\left(x_{0}\right)\right|>1-\frac{\varepsilon^{2}}{4} .
$$

If we normalize we still have

$$
1 \geq \frac{\left|x_{0}^{*}\left(x_{0}\right)\right|}{\left\|x_{0}^{*}\right\|} \geq\left|x_{0}^{*}\left(x_{0}\right)\right| \geq 1-\frac{\varepsilon^{2}}{4} .
$$


Then by applying Remark 2.2, we obtain $y^{*} \in S_{X^{*}}$ and $u_{0} \in S_{X}$ with $\left|y^{*}\left(u_{0}\right)\right|=1$ such that

$$
\left\|x_{0}-u_{0}\right\|<\varepsilon \text { and }\left\|\frac{x_{0}^{*}}{\left\|x_{0}^{*}\right\|}-y^{*}\right\|<\varepsilon
$$

Let $z^{*} \in U \cap M$ be an arbitrary element. Then,

$$
\begin{aligned}
&\left\|z^{*}-y^{*}\right\| \leq\left\|z^{*}-x_{0}^{*}\right\|+\left\|x_{0}^{*}-\frac{x_{0}^{*}}{\left\|x_{0}^{*}\right\|}\right\|+\left\|\frac{x_{0}^{*}}{\left\|x_{0}^{*}\right\|}-y^{*}\right\| \\
& \stackrel{\text { (2.1), (2.3) }}{\leq} \varepsilon+\left\|x_{0}^{*}\right\|\left|1-\frac{1}{\left\|x_{0}^{*}\right\|}\right|+\varepsilon \stackrel{\stackrel{(2.2)}{\leq} 3 \varepsilon,}{\leq},
\end{aligned}
$$

and the proof is over.

Theorem 2.4. Let $T: X \rightarrow C_{0}(L)$ be an Asplund operator with $\|T\|=1$. Suppose that $\frac{1}{2}>\varepsilon>0$ and $x_{0} \in S_{X}$ are such that

$$
\left\|T\left(x_{0}\right)\right\|>1-\frac{\varepsilon^{2}}{4}
$$

Then there are $u_{0} \in S_{X}$ and an Asplund operator $S \in S_{L\left(X, C_{0}(L)\right)}$ satisfying

$$
\left\|S\left(u_{0}\right)\right\|=1,\left\|x_{0}-u_{0}\right\|<\varepsilon \text { and }\|T-S\| \leq 3 \varepsilon .
$$

Proof. The natural embedding $\xi: L \rightarrow C_{0}(L)^{*}$ given by $\xi(s):=\delta_{s}$, for $s \in L$, is continuous for the topology of $L$ and the $w^{*}$-topology in $C_{0}(L)^{*}$. Hence the composition $\phi:=T^{*} \circ \xi: L \rightarrow X^{*}$ is continuous for the $w^{*}$-topology in $X^{*}$.

Now apply Lemma 2.3 for $Y:=C_{0}(L), B:=\left\{\delta_{s}: s \in L\right\} \subset B_{C_{0}(L)^{*}}$, our given operator $T$, and $\varepsilon$. We produce the $w^{*}$-open set $U$ and the functional $y^{*} \in S_{X^{*}}$ satisfying properties (a) and (b) in the aforesaid lemma. Note that with our new notation we have $\phi(L)=M$. Since $U \cap M \neq \emptyset$ we can pick $s_{0} \in L$ such that $\phi\left(s_{0}\right) \in U$. The $w^{*}$-continuity of $\phi$ ensures that the set $W=\{s \in L: \phi(s) \in U\}$ is an open neighborhood of $s_{0}$. By Urysohn's lemma, [17, Lemma 2.12], we can find a continuous function $f: L \rightarrow[0,1]$ with compact support satisfying:

$$
f\left(s_{0}\right)=1 \text { and } \operatorname{supp}(f) \subset W .
$$

Now define the linear operator $S: X \rightarrow C_{0}(L)$ by the formula

$$
S(x)(s)=f(s) \cdot y^{*}(x)+(1-f(s)) \cdot T(x)(s) .
$$

It is easily checked that $S$ is well-defined and that $\|S\| \leq 1$. On the other hand, $1=\left|y^{*}\left(u_{0}\right)\right|=\left|S\left(u_{0}\right)\left(s_{0}\right)\right| \leq\left\|S\left(u_{0}\right)\right\| \leq 1$ and therefore $S$ attains the norm at the point $u_{0} \in S_{X}$ for which we had $\left\|u_{0}-x_{0}\right\|<\varepsilon$.

Now, bearing in mind (2.4), (2.5), Lemma 2.3 and the definition of $W$ we conclude that

$$
\begin{aligned}
\|T-S\| & =\sup _{x \in B_{X}}\|T x-S x\|=\sup _{x \in B_{X}} \sup _{s \in L} f(s)\left|T(x)(s)-y^{*}(x)\right| \\
& =\sup _{x \in B_{X}} \sup _{s \in W} f(s)\left|\phi(s)(x)-y^{*}(x)\right| \leq \sup _{s \in W} \sup _{x \in B_{X}}\left|\phi(s)(x)-y^{*}(x)\right| \\
& =\sup _{s \in W}\left\|\phi(s)-y^{*}\right\| \leq 3 \varepsilon .
\end{aligned}
$$

To finish we prove that $S$ is also an Asplund operator. This is based on the fact that the family of Asplund operators between Banach spaces is an operator ideal; see [22, Theorem 2.12]. Observe that $S$ appears as the sum of a rank one operator and the operator $x \mapsto(1-f) T(x)$; the latter is the composition of a bounded 
operator from $C_{0}(L)$ into itself with $T$. Therefore $S$ is an Asplund operator and the proof is over.

Recall that an operator ideal $\mathcal{I}$ is a way of assigning to each pair of Banach spaces $(X, Y)$ a linear subspace $\mathcal{I}(X, Y) \subset L(X, Y)$ that contains all finite rank operators from $X$ to $Y$ and satisfies the following property: $T_{2} \circ T \circ T_{1} \in \mathcal{I}(Z, V)$ whenever $T \in \mathcal{I}(X, Y), T_{1} \in L(Z, X)$, and $T_{2} \in L(Y, V)$; see [9, 16].

If we denote by $\mathcal{A}$ the ideal of Asplund operators between Banach spaces, the above theorem applies as well to any subideal $\mathcal{I} \subset \mathcal{A}$.

Corollary 2.5. Let $\mathcal{I} \subset \mathcal{A}$ be an operator ideal. Let $T \in \mathcal{I}\left(X, C_{0}(L)\right)$ with $\|T\|=1$, $\frac{1}{2}>\varepsilon>0$, and $x_{0} \in S_{X}$ be such that

$$
\left\|T\left(x_{0}\right)\right\|>1-\frac{\varepsilon^{2}}{4} .
$$

Then there are $u_{0} \in S_{X}$ and $S \in \mathcal{I}\left(X, C_{0}(L)\right)$ with $\|S\|=1$ satisfying

$$
\left\|S\left(u_{0}\right)\right\|=1,\left\|x_{0}-u_{0}\right\|<\varepsilon \text { and }\|T-S\| \leq 3 \varepsilon .
$$

We should stress that because $\mathcal{W} \subset \mathcal{A}$ (see [7]) the above corollary applies in particular to the ideals of finite rank operators $\mathcal{F}$, compact operators $\mathcal{K}, p$-summing operators $\Pi_{p}$ and of course to the weakly compact operators $\mathcal{W}$ themselves. Results in this vein can be found in the literature for weakly compact operators but with spaces of continuous functions as domain spaces and only for the so-called Bishop-Phelps property: Schachermayer proved (see [19, Theorem B]) that any $T \in \mathcal{W}(C(K), X)$ can be approximated by norm-attaining operators. (This result was generalized later for operators $T \in \mathcal{W}\left(C_{0}(L), X\right)$; see [2].) With spaces of continuous functions in the range, Johnson and Wolfe, see [11, Theorem 3], proved that any $T \in \mathcal{K}(X, C(K))$ can be approximated by finite rank norm-attaining operators. Note then that our Corollary 2.5] adds several new versions of the vectorvalued Bishop-Phelps theorem. Moreover, these cases provide the Bollobás part of approximation of points at which the norm is attained.

Standard $\varepsilon-\delta$ tricks suffice to prove that for a pair of Banach spaces $(X, Y)$ the following are equivalent:

(i) $(X, Y)$ has BPBP according to Definition [1.1;

(ii) there are functions $\eta:(0,+\infty) \rightarrow(0,1), \beta, \gamma:(0,+\infty) \rightarrow(0,+\infty)$ with $\lim _{t \rightarrow 0} \beta(t)=\lim _{t \rightarrow 0} \gamma(t)=0$, such that given $\varepsilon>0$, for all $T \in S_{L(X, Y)}$, if $x_{0} \in S_{X}$ is such that $\left\|T\left(x_{0}\right)\right\|>1-\eta(\varepsilon)$, then there exist a point $u_{0} \in S_{X}$ and $S \in S_{L(X, Y)}$ satisfying

$$
\left\|S\left(u_{0}\right)\right\|=1,\left\|x_{0}-u_{0}\right\|<\beta(\varepsilon) \text { and }\|T-S\|<\gamma(\varepsilon) .
$$

Once again, in (ii) above we can always take $\beta(t)=\gamma(t)=t$, but of course changing $\eta$ if needed! Consequently we arrive at the following straightforward consequence of Theorem 2.4 .

Corollary 2.6. For any Asplund space $X$ and any locally compact Hausdorff topological space $L$ the pair $\left(X, C_{0}(L)\right)$ has the $B P B P$. 
Note that this corollary extends and strengthens Theorem 2 in [11; we stress also that we can take as $X$ any $c_{0}(\Gamma)$ ( $\Gamma$ an arbitrary set), or more generally any $C_{0}(S)$, where $S$ is a scattered locally compact Hausdorff space (see, for instance, [15] for scattered or dispersed spaces). Indeed for a locally compact space $S$, the space $C_{0}(S)$ is Asplund if, and only if, $S$ is scattered. This can be proved in the following way:

(1) It is known that for $K$ compact, $C(K)$ is Asplund if, and only if, $K$ is scattered; combine [15, Main Theorem] with Theorem 2.1 or, alternatively, see [14, Theorem 18].

(2) It is easy to check that if $S$ is locally compact, then $S$ is scattered if, and only if, its Alexandroff compactification $S \cup\{\infty\}$ is scattered,

(3) Now use that Asplundness is a three space property (see [14, Theorems 11, 12 and 14]) and conclude that $C_{0}(S)$ is Asplund if, and only if, $C(S \cup\{\infty\})$ is Asplund.

(4) Summarizing, $C_{0}(S)$ is Asplund if, and only if, $S$ is scattered.

Note that whereas the hypothesis of $X$ being Asplund in the above corollary is an isomorphic property, for the range space we have to use the sup norm in $C_{0}(L)$. Indeed, Lindenstrauss [12, Proposition 4] established that if $\left(c_{0},\|\cdot\|\right)$ is a strictly convex renorming of $c_{0}$, then $i d: c_{0} \rightarrow\left(c_{0},\|\cdot\|\right)$ cannot be approximated by normattaining operators. Notice also that Corollary 2.6 may fail when $X$ is not Asplund: Schachermayer [19] gave an example of an operator $T \in L\left(L^{1}[0,1], C[0,1]\right)$ that cannot be approximated by norm-attaining operators.

With our comments above together with Theorem 2.4 we have:

Corollary 2.7. For any Banach space $X$ and any scattered locally compact Hausdorff topological space $L$ the pair $\left(X, C_{0}(L)\right)$ has the BPBP.

An alternative proof for this corollary can be obtained using the fact that for such an $L$ the space $Y=C_{0}(L)$ has property $(\beta)$, see [18, Definition 1.2], and for spaces $Y$ with property $(\beta)$, every pair $(X, Y)$ has BPBP; see [1, Theorem 2.2].

In a different line of ideas, and to finish the paper, we point out that Lindenstrauss proved in [12, Theorem 1] that every operator $T \in L(X, Y)$ can be approximated by operators $S \in L(X, Y)$ such that $S^{* *} \in L\left(X^{* *}, Y^{* *}\right)$ attains the norm on $B_{X^{* *}}$. In [1, Example 6.3] it is established that the counterpart of the above Lindenstrauss result is no longer valid for the corresponding natural BishopPhelps-Bollobás with bi-adjoint operators. The example again uses $c_{0}$ as a domain space. Replacing $Y^{* *}$ by $C\left(B_{Y^{*}}, w^{*}\right)$, we state our last result.

Corollary 2.8. Let $T: X \rightarrow Y$ be an Asplund operator with $\|T\|=1, \quad \frac{1}{2}>\varepsilon>0$ and $x_{0} \in S_{X}$ such that

$$
\left\|T\left(x_{0}\right)\right\|>1-\frac{\varepsilon^{2}}{4} .
$$

Then there are $u_{0} \in S_{X}$ and an Asplund operator $S \in S_{L\left(X, C\left(B_{Y^{*}}\right)\right)}$ satisfying

$$
\left\|S\left(u_{0}\right)\right\|=1,\left\|x_{0}-u_{0}\right\|<\varepsilon \text { and }\|i \circ T-S\| \leq 3 \varepsilon,
$$

where $i: Y \hookrightarrow C\left(B_{Y^{*}}\right)$ is the natural embedding.

\section{ACKNOWLEDGEMENTS}

We gratefully thank Professor Joe Diestel, who, when listening to a preliminary version of this work, reminded us of the beautiful concept of an Asplund operator. We also thank the referee for several helpful comments. 


\section{REFERENCES}

1. María D. Acosta, Richard M. Aron, Domingo García, and Manuel Maestre, The BishopPhelps-Bollobás theorem for operators, J. Funct. Anal. 254 (2008), no. 11, 2780-2799. MR 2414220 (2009c:46016)

2. J. Alaminos, Y. S. Choi, S. G. Kim, and R. Payá, Norm attaining bilinear forms on spaces of continuous functions, Glasgow Math. J. 40 (1998), no. 3, 359-365. MR1660038(2000d:46030)

3. Edgar Asplund, Fréchet differentiability of convex functions, Acta Math. 121 (1968), 31-47. MR.0231199 (37:6754)

4. Errett Bishop and R. R. Phelps, A proof that every Banach space is subreflexive, Bull. Amer. Math. Soc. 67 (1961), 97-98. MR0123174 (23:A503)

5. Béla Bollobás, An extension to the theorem of Bishop and Phelps, Bull. London Math. Soc. 2 (1970), 181-182. MR0267380 (42:2282)

6. Richard D. Bourgin, Geometric aspects of convex sets with the Radon-Nikodým property, Lecture Notes in Mathematics, vol. 993, Springer-Verlag, Berlin, 1983. MR704815 (85d:46023)

7. W. J. Davis, T. Figiel, W. B. Johnson, and A. Pełczyński, Factoring weakly compact operators, J. Functional Analysis 17 (1974), 311-327. MR0355536 (50:8010)

8. J. Diestel and J. J. Uhl Jr., Vector measures, Mathematical Surveys, vol. 15, American Mathematical Society, Providence, R.I., 1977, with a foreword by B. J. Pettis. MR 56:12216

9. Joe Diestel, Hans Jarchow, and Andrew Tonge, Absolutely summing operators, Cambridge Studies in Advanced Mathematics, vol. 43, Cambridge University Press, Cambridge, 1995. MR.1342297 (96i:46001)

10. G. A. Edgar, Asplund operators and a.e. convergence, J. Multivariate Anal. 10 (1980), no. 3, 460-466. MR.588087 (82f:47053)

11. Jerry Johnson and John Wolfe, Norm attaining operators, Studia Math. 65 (1979), no. 1, 7-19. MR 554537 (81a:47021)

12. Joram Lindenstrauss, On operators which attain their norm, Israel J. Math. 1 (1963), 139148. MR0160094 (28:3308)

13. I. Namioka, Radon-Nikodým compact spaces and fragmentability, Mathematika 34 (1987), no. 2, 258-281. MR89i:46021

14. I. Namioka and R. R. Phelps, Banach spaces which are Asplund spaces, Duke Math. J. 42 (1975), no. 4, 735-750. MR0390721 (52:11544)

15. A. Pełczyński and Z. Semadeni, Spaces of continuous functions. III. Spaces $C(\Omega)$ for $\Omega$ without perfect subsets, Studia Math. 18 (1959), 211-222. MR0107806 (21:6528)

16. Albrecht Pietsch, Operator ideals, North-Holland Mathematical Library, vol. 20, NorthHolland Publishing Co., Amsterdam, 1980, translated from the German by the author. MR.582655 (81j:47001)

17. Walter Rudin, Real and complex analysis, third ed., McGraw-Hill Book Co., New York, 1987. MR.924157 (88k:00002)

18. Walter Schachermayer, Norm attaining operators and renormings of Banach spaces, Israel J. Math. 44 (1983), no. 3, 201-212. MR693659 (84e:46012)

19. _ Norm attaining operators on some classical Banach spaces, Pacific J. Math. 105 (1983), no. 2, 427-438. MR691613 (84g:46031)

20. Charles Stegall, The Radon-Nikodym property in conjugate Banach spaces, Trans. Amer. Math. Soc. 206 (1975), 213-223. MR0374381 (51:10581)

21. _ The duality between Asplund spaces and spaces with the Radon-Nikodym property, Israel J. Math. 29 (1978), no. 4, 408-412. MR0493268 (58:12297)

22. Soc. 264 (1981), no. 2, 507-519. MR.603779 (82k:46030)

Department of Mathematical Sciences, Kent State University, Kent, Ohio 44242

E-mail address: aron@math.kent.edu SPAIN

Departamento de Matemáticas, Universidad de Murcia, 30.100 Espinardo, Murcia,

E-mail address: beca@um.es

Department of Mathematical Sciences, Kent State University, Kent, Ohio 44242

E-mail address: okozhush@math.kent.edu 